\title{
Studies on the application of mechanics in aerobics teaching
}

\author{
He Huang \\ Leshan Normal University, Leshan Sichuan, 614000, China
}

Keywords: Aerobics teaching, Mechanics knowledge, Sport injury.

\begin{abstract}
Well received among college students in recent years, aerobics with strong cadence and rhythm is very popular among students in PE classes. Through a brief analysis on mechanical principle of aerobic movements and its application in college aerobics teaching, this article has studied the current development status of aerobics, key points of sports biomechanics in aerobics teaching and biomechanical analysis in sport injury. This article has studied the application of mechanics in aerobics from different aspects, made conclusion and summary, and provided scientific theoretical basis for better developing aerobics sports with the hope that students can have a proficient mastery of the mechanics principles while practicing aerobics for laying foundation for lifelong exercise and study.
\end{abstract}

\section{Introduction}

With social development and economic demands, talent market has higher requirements for students. Cultivating professional, high-quality, physically healthy students have become a major target of teaching and a new requirement for teachers. Under the influence of social environment, teachers' teaching method reform and education method adapting to modern society are issues in front of us. Aerobics is a sports item highly praised by people. College aerobics courses have been promoted by a lot of college students after implementing "three independent"elective system. Particularly for girls, proportion of girls who choose aerobics has reached $30 \%$. Then, aerobics teaching in colleges and universities have entered into a new stage and new platform for development. Classical mechanics and mechanics of materials are the foundation of aerobics sports. A good mastery of mechanical knowledge is of great significance for aerobics learning. The biological foundation of aerobics include the structure and function of human organ system. Studying and learning human structure and organization function is helpful for carrying out the sports and injury protection work. Teachers can help students to better do aerobics movements of they analyze relevant mechanical knowledge at class. Through researches on aerobic movements, students' physical theory and PE theoretical knowledge can be increased. Thus in aerobic teaching, we do not only explain and demonstrate aerobic movements, but combine technical structure of aerobics with the characteristics of biomechanics, combine physical subject knowledge with PE subject knowledge so that students can better master aerobics.

\section{Biomechanical studies on current college aerobics and the current application status}

\section{Current aerobics teaching situation in regular institutions of higher learning}

At present, aerobic teaching methods in Chinese colleges and universities are single and outdated, and students mechanically learn movements demonstrated by teachers so that the overall aerobic strength cannot be improved. In actual teaching, teachers mainly use demonstration teaching method, i.e. students just imitate the movements demonstrated by teachers with no need to think. Under such a teaching method, students can only memorize aerobic movements by temporary memories but will forget after a long time, let alone to innovate the aerobic movements. In PE theoretical education, to a large degree, teachers only let students passively receive and mechanically memorize. Investigations 
show that currently, PE teaching period is about 36 class hours for a semester in most colleges. There are other problems such as physical fitness tests, item evaluation and collision with holidays and festivals. Thus the class hour of theoretical knowledge of aerobics course is only 2 class hours. Few teaching practice in theoretical teaching contents arrangement is almost an issue can never be ignored in PE teaching. Some PE teachers think the class instruction period is short and non-PE specialty students think it not necessary to learn theoretical knowledge. Some students agree with the view thus they don't learn carefully in the rare PE theoretical courses. These are not correct. Secondly, lack of correct theoretical guidance will influence the effect of PE exercise and will result in exercise injury. It can be seen that teachers must attach great importance to mechanics theory teaching in aerobics, especially strengthening the integrated learning of sport dynamics, sport medicine and physical education.

\section{Studies on sports biomechanics and current application status}

Sports biomechanics is a sport science field that applies the laws of mechanics and physics to human performance and is quantitative based study and analysis of professional athletes and sports' activities in general. Its major function is to confirm the action principle of each sports item and it is also the reference basis of whether athletes' skills and training methods are correct or not.

From the perspective of research method, sports biomechanics is categorized into theoretical research method and experiment research method. The former is mainly to explore rules of human motion so as to acquire human movement methods. The most principal research method is human motion mathematical model method. Experiment research method is to use computer technology, advanced experiment methods in medicine and biology to study human body in motion. Studying level and technical analysis degree during the research process depend on the progress of research instrument, meanwhile, synchronization, computerization and stroking technology optimization are required. Theoretical research focuses more on conciseness and accuracy of calculation method.

It can be seen that sports biomechanics is not only an exclusive science in sports field, a large amount of researches have helped its rapid development. For a long time in the past, sports biomechanics was used for sports game research which has played an important role in sports development. Sports biomechanics is a new momentum of promoting the development and progress of sports game, as well as the important support to further promote mass sports activities. It has great research value and development prospect, and great significance for students PE teaching in the future.

\section{Application of sports biomechanics in aerobics teaching}

In recent years, aerobics has developed rapidly in China. But as a subject, theoretical research on it in China is not satisfactory especially the study on sports biomechanics is lagged behind. Aerobics almost cover several kinds of movements, rotation, falling, jumping, body bending, and body swinging. Theoretical principles of these movements come from sports biomechanics theory. But the mechanics application in aerobics is mainly reflected in static and exertive movements, moving movements, thus we should pay additional attention to the mechanics research and instruction of these movements in aerobics teaching so as to obtain the motion rules of aerobics and promote the development of aerobics.

\section{Application of static and exertive movements in aerobics teaching}

Static and exertive movements are research part in statics which firstly studies the balance condition of force and stability of equilibrium. It also involves an issue of stability of equilibrium in aerobics, the mechanical system in equilibrium position can still keep the feature of equilibrium under the influence and even intervention of exterior force. In static movements, the condition of body equilibrium is when the resultant of all the external forces and the resultant moment force are zero at the same time, which means the object is under the resultant of all the external forces and the resultant moment force of zero. For human body, resultant force of zero means that the resultant force of force, moment force and force couple is zero.

In the teaching process, we need to pay attention to explaining equilibrium of forces so that they can obtain the essential theory of movements and biological knowledge and movement standard to 
better complete movements. After acquiring mechanics principles in static and exertive movements, we can help students learn how to improve their own equilibrium and how to maintain the stability of equilibrium through theoretical knowledge instruction. Firstly, make use of the bearing surface, and guide students to notice that the larger area of human body contacting or supporting to the floor, the better people's stability of equilibrium and firmer of their movements, which should be repeatedly instructed; secondly, explain and demonstrate the height of center of body gravity, and demonstrate students through movement analysis that the lower of the center of gravity, the more effective of the overall stability. And the larger of stability angle, the better of the stability; in addition, from the perspective of mechanics, the equilibrium of aerobics movement will be influenced by students' weight. Research data show that the stability of movement has a positive correlation with students' weight.

\section{Application of motility movements in aerobics}

Jumping movements in Aerobics can be divided into take-off, spring-up, and falling. Aerobics almost cover several kinds of movements, rotation, falling, jumping, body bending, and body swinging. Rotation movements are based on the human body vertical axis to achieve the purpose of reducing rotational inertia and increasing angular speed by reducing rotational radius, which can help for body rotation and perfect movement display.

Spring-up movements are on the condition of rotation. When spring up, students should regulate their center of gravity down and jump with the help of the applied force from the floor at the same time displaying body movements. As the height of spring-up is proportional to the original speed, it is necessary to lower the center of gravity so as to improve students' spring-up speed. Thus, during instruction, teachers should remind students of keeping a certain change range of center of gravity and take-off speed for perfect display of aerobic movements.

The energy of aerobic athletes when springing up changes as below: if their center-of-gravity position is a potential energy surface, human chemical energy works during the take-off and pedaling and transform into kinetic energy and gravitational potential energy [1]. Rotation movement is one major movements in aerobics sports. Thus teachers should analyze movements for students and include angular speed, angular accelerated speed, law of rotation and other rules into course teaching so that students can maintain good movement and gesture in rotation process, and reduce radius of rotation, rotational inertia, and increase angular speed so as to display beautiful posture and accelerate rotation speed. In the falling period, the key point of movements is the buffering force when human body contacts with the floor in a high speed. Relevant mechanics knowledge involves the law of impulse, which means it requires to extend the contact time with the acting surface to reduce the impact force of hum body with the floor. In this way, the impact force to human body will greatly reduce. Leg swinging movements are mainly displayed by leg swinging and demonstration of rotation movements. In this kind of movements, swinging amplitude is the basic reference point. When proceeding the movement, the height of leg, rotatory inertia will influence the amplitude of movement and dynamic equilibrium of body. Compared with other movements, there are few bending movements, mainly involving conservation of momentum, law of momentum transfer, mutual relation between human parts and other mechanics theoretical principles.

These moments are based on relevant mechanics principles according to some rules. Thus in actual application, we need to strengthen the instruction of mechanics knowledge and grasp the essential rules of sports through comprehensive and in-depth research to teach mechanics knowledge to students and help them better comprehend aerobic movements.

\section{Biomechanical analysis on sport injury}

Sport injury refer to damage to human body part due to the sproter's personal subjective factors or exterior objective factors. During the course teaching process, some students will easily get sport injuries if paying no attention or not carefully learning theoretical knowledge. Especially in classroom teaching, in front of a lot of students, teachers cannot keep a close eye on each student so that they are very likely to get sport injuries. There are more acute injuries than chronic injuries with 
worse consequences. Inappropriate acute injury treating will result into chronic injuries leaving an incompletely cured illness for a long time so as to affect students' normal life. Thus teachers should pay more attention to students' movement direction in the teaching process.

According to investigations, among 109 mass aerobics learners in a club, the proportion of sports injury takes up 58.7\%, injuries most in joint sprain, shank periostitis, ligament injury, pulled muscle, and exercise induced abdominal pain; acute injuries account for $70.54 \%$, chronic injuries account for $24.1 \%$ and acute injuries into chronic injures account for $14.29 \%{ }^{[2]}$. In aerobics teaching is mainly based on music accompaniment to frequently and powerfully complete different jumping, supporting and rotation, stretching and other single or compound movements. This kind of multi-part movements will easily result in sports injuries. Joint injury is one the most frequently happened. Human articular bone is made by thighbone, tibia, patella, and fibula. It shows the characteristic of long lever up and down and few muscle around, thus it is the most vulnerable body part to sport injury.

Reasons of sports injuries include a lot. Students easily ignore the study of mechanics theoretical knowledge so that they lack effective understanding of sport injury and cannot take necessary measures to prevent sports injury. In addition to the large number of aerobics classes in colleges, individual differences between students, poor health condition of specific students, or students' distraction in class teaching, it easily results in sport injury. Thus, in reaching process, we should pay attention to teaching guidance, and use mechanics knowledge to guide students to avoid sports injury.

\section{Conclusion}

Sports biomechanics application in aerobics can improve students' aerobics professional level and can be very promising in promoting the development of aerobics skill and applying in human health research. Studies on sports biomechanics application in aerobics and scientific design of movements and contents in aerobics can satisfy people's exercise demands and help aerobics teaching in accordance with the reality, and further promote the development of aerobics in China.

\section{Reference}

[1] Li Ling. Application of mechanics principle in aerobics, Participation Education in Middle School Physics, 2015, 12, pp.68-69.

[2] Shi Hongmei. Reasons of sport injuries in aerobics teaching and relevant prevention, Value Engineering, 2012, 31, pp.326-327.

[3] Wang Rui. Brief thinking on aerobics teaching reform in regular colleges and universities, Science \& Technology of Stationary \& Sporting Goods, 2015,18, pp.92-92.

[4] Xiong Haoran, Zhao Li. Brief analysis on the application of mechanics in aerobics, Physics Teaching in Middle School, 2015,7, pp.25-26.

[5] Huang Xinru, Sun Bin, Sun Tingting. Research status of aerobics in biomechanics domain, Sports Time, 2015, 15, pp.16-17.

[6] Wu Shengkou, Ji Zhongqiu. Biomechanical analysis on aerobics athlete's one-time jumping and continuous jumping ability, Journal of Physical Educaion, 2014,3, pp.132-136. 